\title{
Life-threatening pulmonary interstitial lung disease complicating pediatric non-humoral immunodeficiencies
}

DOI:

10.1016/j.jaip.2019.03.034

\section{Document Version}

Accepted author manuscript

Link to publication record in Manchester Research Explorer

\section{Citation for published version (APA):}

Al Farsi, T., Hughes, S. M., Wynn, R., Cheesman, E., Rieux-Laucat, F., Latour, S., Picard, C., Hambleton, S., \& Arkwright, P. (2019). Life-threatening pulmonary interstitial lung disease complicating pediatric non-humoral immunodeficiencies. The Journal of Allergy and Clinical Immunology: In Practice.

https://doi.org/10.1016/j.jaip.2019.03.034

Published in:

The Journal of Allergy and Clinical Immunology: In Practice

\section{Citing this paper}

Please note that where the full-text provided on Manchester Research Explorer is the Author Accepted Manuscript or Proof version this may differ from the final Published version. If citing, it is advised that you check and use the publisher's definitive version.

\section{General rights}

Copyright and moral rights for the publications made accessible in the Research Explorer are retained by the authors and/or other copyright owners and it is a condition of accessing publications that users recognise and abide by the legal requirements associated with these rights.

\section{Takedown policy}

If you believe that this document breaches copyright please refer to the University of Manchester's Takedown Procedures [http://man.ac.uk/04Y6Bo] or contact uml.scholarlycommunications@manchester.ac.uk providing relevant details, so we can investigate your claim.

\section{OPEN ACCESS}




\section{The Journal of Allergy and Clinical Immunology: In Practice}

\section{Life-threatening pulmonary interstitial lung disease complicating pediatric non-humoral immunodeficiencies \\ --Manuscript Draft--}

\begin{tabular}{|l|l|}
\hline Manuscript Number: & INPRACTICE-D-19-00055R1 \\
\hline Article Type: & Clinical Communication (Brief report) \\
\hline Section/Category: & Immune Deficiencies, Infection, and Systemic Immune Disorders \\
\hline Keywords: & primary immunodeficiency diseases; children; interstitial lung disease; GLILD; HSCT \\
\hline Corresponding Author: & $\begin{array}{l}\text { Peter D Arkwright, MB, D Phil } \\
\text { University of Manchester } \\
\text { Manchester, Lancashire UNITED KINGDOM }\end{array}$ \\
\hline First Author: & Tariq Al Farsi, MD \\
\hline Order of Authors: & Tariq Al Farsi, MD \\
\hline Stephen M Hughes \\
\hline Robert F. Wynn, MD \\
\hline Edmund Cheesman, MD \\
\hline Frederic Rieux-Laucat, PhD \\
\hline Sylvain Latour, PhD \\
\hline Capucine Picard, MD, PhD \\
\hline Sophie Hambleton, MD, PhD \\
\hline Peter D Arkwright, MB, D Phil \\
\hline UNITED KINGDOM \\
\hline Manuscript Region of Origin: & $\begin{array}{l}\text { Clinical Implications: Interstitial Lung Disease (ILD) in children often indicates life- } \\
\text { threatening PID and has a poor prognosis without hematopoietic stem cell } \\
\text { transplantation. Lung biopsy often provides definitive pathological results that help to } \\
\text { direct management decisions. }\end{array}$ \\
\hline &
\end{tabular}




\section{INPRACTICE-D-19-00055, Life-threatening pulmonary interstitial lung disease complicating pediatric non-humoral immunodeficiencies}

\section{POINT BY POINT RESPONSE}

\section{COMMENTS FROM REVIEWER \#1:}

In this case series, the authors present the details of 9 pediatric patients with PIDD and ILD, screened from a cohort of 1034 pediatric referrals to their PIDD clinic. This adds to the literature since little has been published on this topic to date specific to the pediatric population.

Comment: Page 4, Line 61 - Suggest to highlight here the important observation that in this cohort, in all but two cases, the age of onset of chest symptoms preceded the diagnosis of lung disease, which in turn preceded the diagnosis of PIDD.

Response: As suggested, a sentence highlighting this important point as been added to the text.

Page 4 Line 78 - Would highlight that the lack of observed granulomata is in definite contrast to the granulomatous inflammation seen in GL-ILD associated with CVID.

Response: The referee raises a good point. Contrary to what might be assumed from the descriptive term, GLILD is not usually associated with granuloma, but rather more diffuse collections of histiocytes and lymphocytes. This point has now been highlighted and referenced in the text.

Comment: Page 5 Last sentence - There appears to be a missing word "HSCT can be life-saving but should be carried out by experienced transplant physicians as post-transplant clinical course, as severe inflammation of the lungs, liver and gut are common in this group of patients."

Response: The sentence has now been rephrased.

Table I - the IgG levels are relatively high in most cases, please clarify if this is reactive or if patients were on supplemental immunoglobulin?

Response: None of the patients were on immunoglobulins replacement, those with high IgG were reactive. The fact that some patients have high reactive IgG are detailed in the Supplementary case histories.

Comment: Supplement Page 14 Line 26 - typo in word "splice"

Response: Typo corrected.

\section{COMMENTS FROM REVIEWER \#2:}

This is important and helpful information in an unusual complication of a rare disease. It is first to describe interstitial lung diseases in relation to phenotype in children such detail so it is just a pity that it is a short communication but even so - it will be useful. The cases are particularly helpful and interesting - this should be encouraged in this journal when PIDs (which are rare) are discussed.

I have a few suggestions

Comment: * CVID was designated Common Variable Immunodeficiency Disorders by the IUIS in 2009 - as this is a diagnosis of exclusion

Response: The full expanded name of the disorder has now been added.

* GLILD -this term is confusing and most clinical immunologists are trying to avoid using it Response: The authors fully agree with the reviewer's comment, and have kept the use of term GLILD to a minimum in this manuscript - using it only two in the manuscript. 
Comment: * It is not clear what roles of the non-Manchester authors played; presumably in diagnosis of each case but this should be stated.

Response: Professor Hambleton, although based in Newcastle upon Tyne, does regular joint pediatric immunology outpatient clinics in Manchester to provide advice and input on our complex patients - her affiliation with the Department of Paediatric Allergy and Immunology in Manchester has now detailed in the author affiliation list. Colleagues (Latour, Picard and Rieux-Laucat) from the Imagine Institute in Paris provided important input, determining the definitive genetic diagnosis of a number of the patients in this report. This information has now been added to the acknowledgement section.

Comment: * Given that CVIDs in adults are mentioned early on, it would be helpful to compare the findings with those reported in these adults - some CVID patients have combined immune deficiencies, much like children.

Response: Some of the histological features (particularly the lack of well-formed granuloma) of GLILD in adults with CVID have now been compared with the histological results in this cohort (see response to second point by referee 1 ).

Comment: * A stronger statement about survival in relation to HSCT would be helpful too Response: As recommended, the statement about survival and outcome in relation to HSCT has been strengthened (second last line of text). It has also been highlighted in the "Clinical implications" paragraph after the beginning of the text.

Comment: * Were the follicles described in patients 2 and $4 \mathrm{~B}$ cell follicles with GC or was there no immunochemistry?

Response: In both patient 2 and 4, there were some B-cell follicles which stained with CD20, as shown in Figure 1 (CGD and LRBA respectively.

\section{COMMENTS FROM THE EDITORIAL OFFICE:}

Comment: ${ }^{* *}$ Please remove the list of key words from the manuscript.

Response: As requested, key word list has now been removed.

Comment: ** Please remove the closure (listing of authors at the end of the manuscript text body). Response: List of authors at end of manuscript removed as requested.

Comment: ** Replace the Highlights Box with the required Clinical Implications statement: 1-2 sentences (maximum 40 words) that summarize the clinical implications and importance of the report.

Response: Highlights Box replaced with Clinical Implications statement as requested.

Comment: ** On the title page, please include a conflict of interest statement disclosing all financial and organizational relationships for ALL authors of the manuscript. Authors should disclose all potential conflicts of interest including funding sources that supported their work and any commercial and/or organizational associations. These include consultant arrangements, speakers' bureau participation, stock or other equity ownership, patent licensing arrangements, support such as financial or materials grants for research, employment, or expert witness testimony. If an author has no conflicts to disclose, this should be included in the statement. Further information can be found at http://www.elsevier.com/conflictsofinterest and at http://service.elsevier.com/app/answers/detail/a id/286/supporthub/publishing. This statement should be viewed and approved by all authors.

Response: A conflict of interest statement has now been included on the front page of the manuscript. 

1 Life-threatening pulmonary interstitial lung disease complicating pediatric non-

2 humoral immunodeficiencies

3

$4 \quad$ Tariq Al Farsi ${ }^{1}$ MD, Stephen M Hughes ${ }^{1} M B$ PhD, Robert F. Wynn ${ }^{2}$ MD, Edmund

5 Cheesman $^{3}$ MD, Frederic Rieux-Laucat ${ }^{4}$ PhD, Sylvain Latour ${ }^{4}$ PhD, Capucine Picard ${ }^{4,5}$ MD,

$6 \quad$ PhD, Sophie Hambleton ${ }^{61,6}$ MD, PhD, Peter D. Arkwright ${ }^{1}$ MB D Phil

7

$8{ }^{1}$ University of Manchester, Department of Paediatric Allergy \& Immunology, Manchester,

9 United Kingdom; ${ }^{2}$ University of Manchester, Department of Paediatric Haematology,

$10{ }^{3}$ Department of Paediatric Histopathology, Royal Manchester Children's Hospital,

11 Manchester, United Kingdom; ${ }^{4}$ Imagine Institute, Immunology, Paris, France; ${ }^{5}$ Study Center

12 for Primary Immunodeficiencies, Necker-Enfants Malades Hospital, APHP, Paris, France;

$13{ }^{6}$ University of Newcastle, Newcastle upon Tyne, United Kingdom

Author for correspondence: Dr P D Arkwright, Senior Lecturer in Paediatric Immunology,

Department of Paediatric Allergy \& Immunology, Royal Manchester Children’s Hospital,

Oxford Rd., Manchester, M13 9WL, United Kingdom, Tel + 44161701 0678, email

peter.arkwright@nhs.net

20 There were no external sources of funding. None of the authors declare any conflict of

21 interests in relation to this study.

22 Key words: primary immunodeficiency disease, children, interstitial lung disease, GLILD

23 HSCT 
25 Clinical Implications: Interstitial Lung Disease (ILD) in children often indicates life-

26 threatening PID and has a poor prognosis without hematopoietic stem cell transplantation.

1. What is already known about this topic? Interstitial Lung Disease (ILD) occurs in 20\% of adults with CVID, usually of unclear genetic etiology. In children, there are only a few reports linking ILD to specific primary immunodeficiency diseases (PID).

27 Lung biopsy often provides definitive pathological results that help to direct management

28 decisions. 
To the Editor,

Recurrent acute and chronic lung infections associated with bronchiectasis are a major cause of morbidity and mortality in patients with Primary Immunodeficiency Disorders (PID).${ }^{1-3}$ In adults, inflammatory Interstitial Lung Disease (ILD) not due to pyogenic infection is found in up to $20 \%$ of patients with Common Variable Immunodeficiency Disorders (CVID) and can be associated with progressive restrictive lung disease and shortened survival. ${ }^{4-5}$ High resolution chest CT scan is the gold standard imaging technique for diagnosing ILD. Where there is doubt about the diagnosis lung biopsy is recommended. ${ }^{1,2}$ Histology classically shows lymphocytic infiltrates and/or granuloma, which in adults with CVID is termed Granulomatous-Lymphocytic Interstitial Lung Disease (GLILD). ${ }^{6}$ Although there are reports of ILD associated with a number of monogenic PIDs including Cytotoxic T-LymphocyteAssociated protein 4 (CTLA4), Lipopolysaccharide (LPS)-Responsive and Beige-like Anchor protein (LRBA), Recombination-Activating Gene (RAG1) deficiencies, X-linked Inhibitor of Apoptosis Protein (XIAP) and Chronic Granulomatous Disease (CGD), ${ }^{2,7,8}$ there are no published series delineating the prevalence, clinical characteristics, histologic features and management of children with PID and ILD. This retrospective case series aimed to review the genotype and clinicopathological phenotype of children presenting to our tertiary pediatric primary immunodeficiency center at Royal Manchester Children's Hospital, Manchester, United Kingdom between 2005 and 2018 with ILD and PID who underwent detailed investigations including chest CT scans and in most cases lung biopsy. We hypothesized that ILD in children often indicates life-threatening primary immunodeficiency disease and has a poor prognosis without hematopoietic stem cell transplantation (HSCT).

The case note review was part of a Natural History and Causes of CVID study approved by the Local Research Ethics Committee (03/ST/016). The demography, immunology, histology and genetic screening of nine patients $(0.9 \%)$ with PID and ILD out 
of 1,034 new pediatric referrals to our service because of recurrent, unusual or serious infections or inflammatory diseases were collated (Table I, Figure 1, and online supplementary material). This series of patients includes all but one patient known to Departments of Paediatric Histopathology, Immunology and Respiratory Medicine who had lung biopsies which demonstrated ILD associated with lymphocytic infiltration and/or granuloma formation.

Seven of the nine patients were males. Seven were Asian. All presented with a chronic cough and breathlessness suggestive of recurrent chest infections or asthma. In all but one case in which there was a family history of PID, it was the onset of chest symptoms that prompted a search for an underlying immunodeficiency disorder. A genetic diagnosis was confirmed in seven patients. Three children had a primary neutrophil immunodeficiency (two p40phox Chronic Granulomatous Disease (CGD) and one G6PC3 deficiency), and four a primary T-cell immunodeficiency (RAG1, STK4, LRBA, ITK). Whole exome sequencing of an eighth patient with pulmonary EBV-driven LPD uncovered a potentially pathogenic variant in a gene involved in T-cell signalling and further experiments to confirm the functional relevance of this novel finding are in progress. The ninth patient died from pulmonary Hemophagocytosis LymphoHistiocytosis (HLH) in 2006. Limited candidate gene screening at the time was negative and there was insufficient DNA available for subsequent whole exome sequencing. The median age of onset of chest symptoms of the nine patients was 12 months old. Except for one male who presented in the second year of life with RAG1 deficient Severe Combined Immunodeficiency (SCID) and died of progressive multifocal leukoencephalopathy, and a teenage boy who died of fulminant pulmonary HLH, the remaining seven patients were alive 12 months to 14 years post-HSCT.

Lung biopsies assisted to direct clinical management (Figure 1). Tuberculosis and fungal infections were excluded, allowing for the discontinuation of anti-microbials. No 
evidence of vasculitis was found in any of the patient's biopsies. Although granulomata were only seen in the patients with CGD, a number of the patients had a prominent histiocytic

(CD68) infiltrate. This is in keeping with the lung histology of GLILD in adults with CVID, which is characterized by loose, more diffuse collections of histiocytic and lymphocytic $\underline{\text { infiltrates rather than well-defined clusters of histiocytes and multinuclear giant cells typical }}$ of sarcoidosis..$^{5}$ In the three patients with primary neutrophil immunodeficiencies and the patient with the LRBA deficiency, lung biopsy confirmed the diagnosis of an inflammatory IBD rather than pyogenic infection, and responded to high dose corticosteroids prior to HSCT. In the four patients with primary T-cell immunodeficiencies, lung biopsies confirmed EBV-driven LPD, allowing focused treatment with rituximab \pm EBV-directed cytotoxic Tcells or chemotherapy. The lung biopsies of the teenager who died showed a mixed histiocytic, T- and B-lymphocytic infiltrate and evidence of hemophagocytosis. Infection, including EBV-driven LPD and vasculitis were excluded.

In conclusion, this is the first case series to delineate the variation in genotype and the frequent life-threatening clinical phenotype of children with ILD complicating PID. Lung biopsy often provides important information that helps to direct management decisions, not only by ruling out fungal and mycobacterial infections and vasculitis, but also in tailoring therapy based on the presence of inflammatory infiltrates or EBV-driven LPD. Diagnosis of the underlying PID is challenging, as patients may not only present with classical phenotypes such as EBV-positive ILD secondary to ITK deficiency, but also with unusual manifestations, for example "late-onset" SCID, or p40phox CGD where the standard dihydrorhodamine test may be normal. ${ }^{8,9}$ HSCT can be life-saving- and should be considered as a definitive, therapeutic option. It is however important that HSCT is but should be carried out by experienced transplant physicians ${ }_{2}$ as the post-transplant elinical course can be complicated by, as-severe inflammation of the lungs, liver and gut are common-in this group of patients. 
$\mid \begin{array}{ll}104 & \\ 105 & \text { Tariq Al Farsi } M D \\ 106 & \text { Stephen M Hughes }{ }^{7} M B P h D \\ 107 & \text { Robert F. Wynn }{ }^{2} \text { MD } \\ 108 & \text { Edmund Cheesman }{ }^{3} \text { MD } \\ 109 & \text { Frederic Rieux Laucat }{ }^{4} \mathrm{PhD} \\ 110 & \text { Sylvain Latour }{ }^{4} \mathrm{PhD} \\ 111 & \text { Capucine Picard }\end{array}$

112 Sophie Hambleton ${ }^{6} \mathrm{MD}, \mathrm{PhD}$

113 Peter D. Arkwright ${ }^{+}$MB D Phit

114

$115{ }^{+}$University of Manchester, Department of Paediatric Allergy \& Immunology, Manchester, 116 United Kingdom; ${ }^{2}$ University of Manchester, Department of Paediatric Haematology, $117{ }^{3}$ Department of Paediatric Histopathology, Royal Manchester Children's Hospital, 118 Manchester, United Kingdom; ${ }^{4}$ Imagine Institute, Immunology, Paris, France; ${ }^{5}$ Study Center 119 for Primary Immunodeficiencies, Necker-Enfants Malades Hospital, APHP, Paris, France; $120{ }^{6}$ University of Neweastle, Neweastle upen Tyne, United Kingdom

\section{Acknowledgements}

122 The authors are grateful to colleagues in the Departments of Histopathology at Great Ormond

123 St Hospital for Sick Children, London and the Royal Brompton Hospital, London for

124 reviewing atypical cases. Drs Latour, Picard and Rieux-Laucat provided the genetic diagnosis

125 for a number of the complex patients described in this report and helped draft the manuscript. 


\section{References}

127

1. Cinetto F, Scarpa R, Rattazzi M, Agostini C. The broad spectrum of lung diseases in primary antibody deficiencies. Eur Respir Rev. 2018;27:149.

2. Baumann U, Routes JM, Soler-Palacín P, Jolles S. The Lung in Primary Immunodeficiencies: New Concepts in Infection and Inflammation. Front Immunol. 2018;9:1837.

3. Jesenak M, Banovcin P, Jesenakova B, Babusikova E. Pulmonary manifestations of primary immunodeficiency disorders in children. Front Pediatr. 2014;2:77.

4. Hartono S, Motosue MS, Khan S, Rodriguez V, Iyer VN, Divekar R, et al. Predictors of granulomatous lymphocytic interstitial lung disease in common variable immunodeficiency. Ann Allergy Asthma Immunol. 2017;118:614-620.

5. Bates CA, Ellison MC, Lynch DA, Cool CD, Brown KK, Routes JM. Granulomatouslymphocytic lung disease shortens survival in common variable immunodeficiency. $\mathbf{J}$ Allergy Clin Immunol. 2004;114:415-21.

6. Hurst JR, Verma N, Lowe D, Baxendale HE, Jolles S, Kelleher P, et al. British Lung Foundation/United Kingdom Primary Immunodeficiency Network Consensus Statement on the Definition, Diagnosis, and Management of Granulomatous-Lymphocytic Interstitial Lung Disease in Common Variable Immunodeficiency Disorders. J Allergy Clin Immunol Pract. 2017;5:938-945.

7. Steele CL, Doré M, Ammann S, Loughrey M, Montero A, Burns SO, et al. X-linked Inhibitor of Apoptosis complicated by Granulomatous Lymphocytic Interstitial Lung Disease (GLILD) and granulomatous hepatitis. J Clin Immunol. 2016;36:733-8.

8. van de Geer A, Nieto-Patlán A, Kuhns DB, Tool AT, Arias AA, Bouaziz M, et al. Inherited p40phox deficiency differs from classic chronic granulomatous disease. J Clin Invest. 2018;128:3957-3975. 
$153 \quad$ Figure Legends

154

155 Figure 1 Characteristic lung biopsies of different PID associated with interstitial lung

156 disease. Panels (top to bottom) show Hematoxylin \& Eosin (H\&E), CD3 (T-cells), CD20 (B-

157 cells), CD68 (histiocytes), EBV in situ hybridization (EBV) for each condition. 


\begin{tabular}{|c|c|c|c|c|c|c|c|c|c|c|c|c|c|c|c|c|c|c|c|c|}
\hline 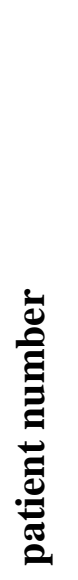 & 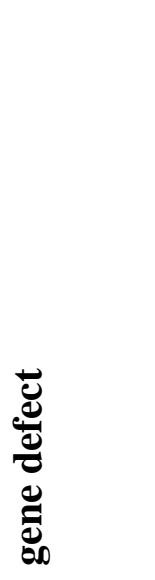 & 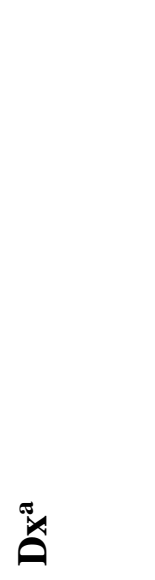 & 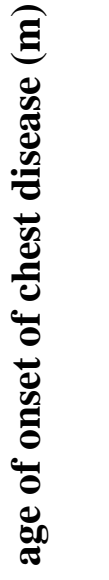 & 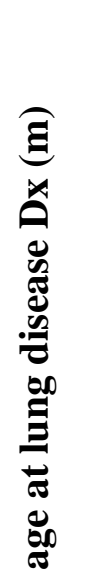 & 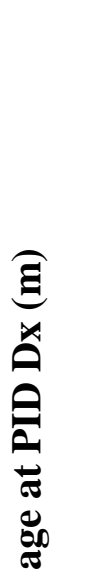 & 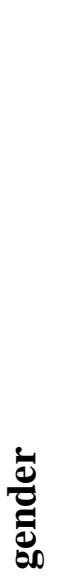 & 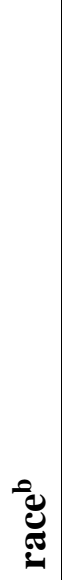 & 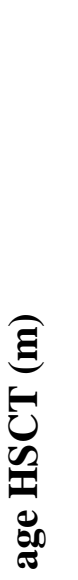 & 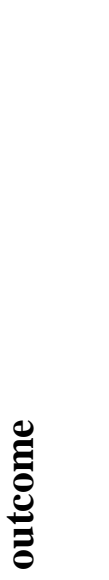 & 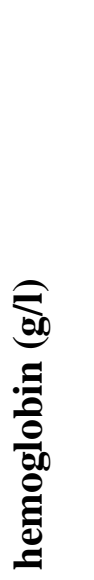 & 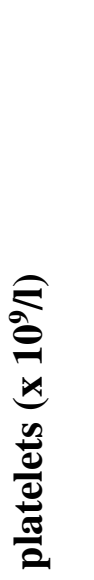 & 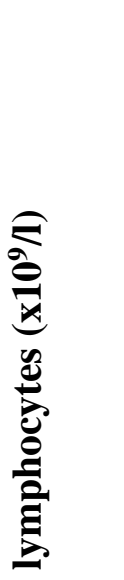 & 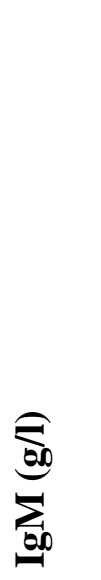 & $\underbrace{\underset{\sigma_{0}}{0}}_{\underbrace{0}_{00}}$ & 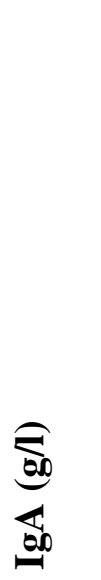 & 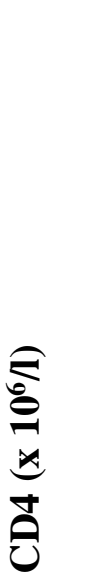 & 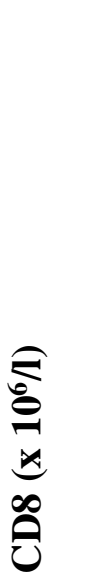 & 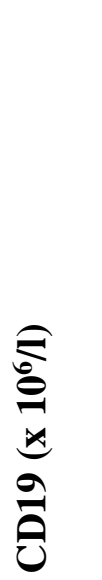 & 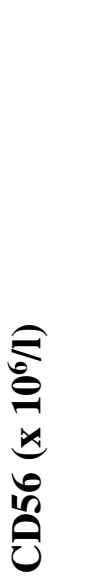 & 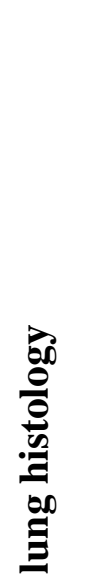 \\
\hline 1 & NCF4 & CGD & 24 & 31 & 36 & $\mathrm{f}$ & A & 56 & alive & 119 & 215 & 3.04 & 1.0 & 12 & 2.1 & 1058 & 933 & 1415 & 129 & G \\
\hline 2 & NCF4 & CGD & 8 & 44 & 56 & $\mathrm{f}$ & $\mathrm{A}$ & 62 & alive & 125 & 297 & 1.88 & 0.98 & 13.8 & 1.76 & 906 & 766 & 3755 & 32 & $\mathrm{~L}$ \\
\hline 3 & G6PC3 & G6PC3 & 1 & 97 & 112 & $\mathrm{~m}$ & A & 142 & alive & 128 & 58 & 0.49 & 0.44 & 17.9 & 0.32 & 339 & 271 & 164 & 23 & $\mathrm{~L}$ \\
\hline 4 & $L R B A$ & LRBA & 3 & 138 & 138 & $\mathrm{~m}$ & $\mathrm{~A}$ & 144 & alive & 80 & 173 & 1.8 & 1.27 & 7.65 & 0.48 & 495 & 282 & 794 & 39 & $\mathrm{~L}$ \\
\hline 5 & ITK & CID & 7 & 10 & 1 & $\mathrm{~m}$ & $\mathrm{~A}$ & 12 & alive & 124 & 351 & 3.11 & 1.32 & 6.6 & 0.87 & 2072 & 978 & 993 & 162 & E-L* \\
\hline 6 & STK4 & CID & 12 & 12 & 142 & $\mathrm{f}$ & $\mathrm{A}$ & 144 & alive & 105 & 348 & 1.30 & 1.06 & 31.1 & 4.89 & 100 & 570 & 270 & 200 & E-L \\
\hline 7 & $R A G 1$ & CID & 14 & 22 & 27 & $\mathrm{~m}$ & $\mathrm{O}$ & - & died & 111 & 510 & 0.17 & 1.56 & 6.82 & $<0.1$ & 19 & 50 & 37 & 221 & E-L \\
\hline 8 & $?$ & CID & 48 & 69 & - & $\mathrm{m}$ & $\mathrm{A}$ & 98 & alive & 138 & 340 & 3.8 & 3 & 21 & 30 & 800 & 1887 & 283 & 69 & $\Phi$ \\
\hline 9 & $?$ & HLH & 168 & 180 & - & $\mathrm{m}$ & $\mathrm{C}$ & - & died & 97 & 60 & 0.41 & 5.02 & 11.2 & 1.22 & 260 & 200 & 60 & 20 & $\mathrm{~L}$ \\
\hline
\end{tabular}


160 Dx: diagnosis, PID: primary immunodeficiency disease, NCF4: Neutrophil Cytosolic Factor 4, ITK: IL-2 Inducible T-Cell Kinase, STK4:

161 Serine/Threonine Kinase 4, RAG1: Recombination Activating Gene 1, a CGD: chronic granulomatous disease, G6PC3: Glucose-6-Phosphatase

162 Catalytic Subunit 3 deficiency, LRBA: lipopolysaccharide (LPS)-responsive and beige-like anchor protein deficiency, CID: combined T/B-cell

163 immunodeficiency, ${ }^{\mathrm{b}} \mathrm{C}$ : Caucasian A: Asian, O: Other. Lung histology: non-caseating granuloma (G), lymphoid hyperplasia (L), lymphoma (L*),

$164 \mathrm{EBV}(\mathrm{E}), \Phi$ - EBV-lymphoproliferative disease diagnosed after finding EBV from sputum, nodular shadowing on CT scan images and response

165 to rituximab. 


\section{Online material}

2 Life-threatening pulmonary interstitial lung disease complicating pediatric non-humoral 3 immunodeficiencies

$4 \quad$ Tariq Al Farsi ${ }^{1}$ MD, Stephen M Hughes ${ }^{1}$ MB PhD, Robert F. Wynn ${ }^{2}$ MD, Edmund Cheesman ${ }^{3}$ 5 MD, Frederic Rieux-Laucat ${ }^{4}$ PhD, Sylvain Latour ${ }^{4}$ PhD, Capucine Picard ${ }^{4,5}$ MD, PhD, Sophie $6 \quad$ Hambleton $^{6}$ MD, PhD, Peter D. Arkwright ${ }^{1}$ MB D Phil

$8 \quad{ }^{1}$ University of Manchester, Department of Paediatric Allergy \& Immunology, Manchester,

9 United Kingdom; ${ }^{2}$ University of Manchester, Department of Paediatric Haematology,

$10{ }^{3}$ Department of Paediatric Histopathology, Royal Manchester Children's Hospital, Manchester, 11 United Kingdom; ${ }^{4}$ Imagine Institute, Immunology, Paris, France; ${ }^{5}$ Study Center for Primary 12 Immunodeficiencies, Necker-Enfants Malades Hospital, APHP, Paris, France; ${ }^{6}$ University of 13 Newcastle, Newcastle upon Tyne, United Kingdom 
Clinical case vignettes (high resolution chest CT scans are shown in Figure S1)

Patient 1 A four-year-old Pakistani girl presented at the age of two years old with a chronic cough and clubbing, associated with cervical lymphadenopathy and hepatosplenomegaly. Parents were first cousins. There was no family history of chest problems or tuberculosis. A chest x-ray showed bilateral, multinodular shadowing. Chest CT scan confirmed patchy infiltrates in the right and left lower lobes, but no bronchiectasis. Hilar lymphadenopathy was noted, particularly on the right $(7 \times 10 \mathrm{~mm})$. Bronchioalveolar lavage revealed no microbes. Lymph node biopsy showed granulomatous inflammation without caseation. Stains for mycobacteria and fungi were negative. Because of the chronic unremitting nature of the lung disease a lung biopsy was performed, which showed granulomatous lymphocytic infiltration with no organisms identified.

Standard dihydrorhodamine test with PMA was normal, but stimulation with E. coli was reduced. Gene sequencing revealed a homozygous c.118-1G>A splice variant in the NCF4 gene consistent with p40phox CGD. ${ }^{8}$ Parents were both heterozygous carriers. The symptoms did not respond to anti-microbials and could only partly be controlled with intermittent pulse intravenous methylprednisolone.

In view of the diagnosis of CGD and her ongoing inflammatory lung disease, she underwent matched-sibling HSCT at the age of three years old. Post-transplant course was complicated by mild veno-occlusive disease treated with defibrotide, followed by fulminant inflammatory disease of her gut, liver and lungs, complicated by pulmonary hemorrhage, requiring high frequency oscillatory ventilation in intensive care. The lung disease was brought under control with anti-TNF therapy and extra-corporal photopheresis. Twelve months posttransplant she developed autoimmune thrombocytopenia, which responded to rituximab. 
Eighteen months post-transplant, her condition is stable and systemic immune suppression has been discontinued. Peripheral leukocytes are $100 \%$ donor.

Patient 2 A seven-year-old Pakistani girl presented with a history of chronic chest symptoms going back to when she was eight months old. She had a chest deformity, clubbing and oxygen saturations in air of $88 \%$. Her growth and development were delayed and she had intermittent fevers and an iron deficiency anemia. Parents were first cousins. No pathogens had been isolated from her chest. Because of wheeze and breathlessness, she had been treated with recurrent courses of oral prednisolone. Chest x-ray and CT scan showed hilar lymphadenopathy and bilateral patchy shadowing and nodules especially in both lower lobes. There was no bronchiectasis. Lung biopsy showed interstitial inflammation with lymphoid follicle formation. There was alveolar remodelling, prominent type 2 pneumocyte hyperplasia and abundant intraalveolar proteinaceous exudates.

Dihydrorhodamine test with PMA was normal, but stimulation with E. coli was reduced. Gene sequencing revealed a homozygous c. $32+2 \mathrm{~T}>\mathrm{G}$ variant in $N C F 4$ consistent with $\mathrm{p} 40 \mathrm{phox}$ CGD. Both parents were found to be carriers. Her inflammatory lung disease was only partly controlled with pulse methylprednisolone therapy. She therefore underwent matched unrelated HSCT at the age of five years old. Three months post-transplantation, she developed lung EBV LPD, which was treated with rituximab. Four months post-transplant she developed a central line infection complicated by Klebsiella pneumoniae septicemia, cardiac tamponade and cardiogenic shock requiring intensive care. Two years post transplantation she is now well and off all medication.

Patient 3 A 12-year-old Pakistani boy suffered from episodes of cough, wheeze and breathlessness from one month of age. He had been treated with multiple courses of antibiotics, inhaled steroids, nebulized bronchodilators and oral prednisolone. He also had chronically loose 
stools. His parents were first cousins. He was referred to our primary immunodeficiency centre at the age of nine years old after moving to the UK. He had been diagnosed with a congenital neutropenia at the age of six years and started on regular G-CSF, although continued to have persistent cough and dyspnea, with poor exercise tolerance requiring supplementary night time oxygen. $\mathrm{FEV}_{1}$ and FVC were $30 \%$ of predicted. Stool culture, bronchoscopy and bronchoalveolar lavage revealed no pathogens. Mantoux and Quantiferon tests were negative.

High resolution chest CT scan showed air trapping and nodular opacities in the left lower lobe, with only mild basal bronchiectasis. Lung biopsy showed peri-bronchial mononuclear infiltrate with occasional lymphoid nodules with no granuloma, fungi or mycobacteria. There was no fibrosis or pulmonary hypertensive changes. Gut biopsies showed inflammation with cryptitis, crypt abscesses, inflammatory infiltrate throughout the colon but no granuloma. There were also patchy inflammatory changes in the esophagus, stomach and terminal ileum.

In view of his congenital neutropenia, symmetrical growth retardation, cryptorchidism and distended veins on his anterior chest, genetic testing was performed which confirmed a homozygous frameshift c882_903 duplication in the G6PC3 gene. Because of frequent hospital admissions for his lung disease and poorly controlled inflammatory bowel disease he underwent matched unrelated HSCT. Fifteen months post-transplant, he is now able to scooter along the flat and not needing supplementary oxygen. His stool frequency has also decreased. Post-transplant immune suppression has been discontinued with $100 \%$ donor engraftment.

Patient 4 An eleven-year-old Indian boy presented in infancy with chronic watery diarrhea due to an autoimmune non-granulomatous colitis. He was treated for four years with corticosteroids, to which azathioprine was added. His parents were first cousins. At seven years old he developed generalized atopic dermatitis and at eight years old cervical lymphadenopathy, splenomegaly, chronic urticaria, a Coombs positive hemolytic anemia and ITP with epistaxis, 
which only partly responded to corticosteroids, methotrexate and IV rituximab. At the age of ten years old, while on the methotrexate (10mg/m²/week), he developed ILD (EBV positive by PCR on sputum but not blood) with fevers and signs of respiratory distress. High resolution chest CT scan showed confluent dense consolidation in both lower lobes, multiple areas of hazy nodular, ground glass changes and hilar lymphadenopathy. Lung biopsy showed a diffuse, reactive lymphoid hyperplasia with lymphoid follicle formation. At the same time his autoimmune cytopenias became more difficult to control. His lung disease and cytopenias only partly responded to rituximab, and required high dose systemic corticosteroids.

Whole exome sequencing revealed a homozygous c.5770_5776 deletion in the LRBA gene. Both parents were carriers. As there were no suitable matched sibling or unrelated donors, he underwent a maternal haploidentical peripheral blood TCR $\alpha \beta$-depleted HSCT with reduced intensity conditioning. His post-transplant course was uncomplicated. Four years posttransplant he off all medication and has $100 \%$ donor engraftment. His chest disease, autoimmune enteropathy and cytopenias have resolved. Because of proteinuria, he investigated and diagnosed with diffuse mesangial sclerosis, which is currently not progressive and he has normal renal function and no proteinuria.

Patient 5 Prompted by a family history, a 15-year-old boy diagnosed with a homozygous c.626G > A ITK deficiency at the age of one month old. HSCT was also delayed because of Hirschsprung's disease requiring surgery in the neonatal period and the difficulty finding a suitable donor. At the age of seven months, EBV viremia was noted and chest CT scan showed widespread bulky masses throughout both lungs. The largest of these replaced most of the right upper lobe. There were multiple smaller nodules scattered throughout both lungs. Lung biopsy showed replacement of normal parenchyma with an EBV-driven diffuse large B-cell lymphoma. He was treated with rituximab and UK CCSG NHL901 chemotherapy protocol, 
followed by a single allelic A mismatched related donor transplant. He remains well off all therapy 14 years post HSCT.

Patient 6 A 16-year-old Asian girl presented at the age of three years old with a history of recurrent infections from 14 months old, when she developed viral croup requiring ventilation and cryptosporidial diarrhea. Over the next few years, she suffered from recurrent Streptococcus pneumoniae pneumonia and ear infections, as well as recurrent thrush, molluscum contagiosum and herpes gingivostomatitis requiring intravenous acyclovir. She had a chronic productive cough, which did not respond to asthma inhalers. Examination revealed tachypnea and oxygen saturations in air of $94 \%$, a discharging left ear and oral thrush.

Investigations showed a CD4 lymphopenia but raised, reactive serum immunoglobulins and protective vaccine antibody responses. HIV screen was negative. EBV was detected in the blood by PCR. Chest CT scan showed multiple small nodules of different sizes throughout both lungs and no bronchiectasis. Lung biopsy showed a B-cell infiltrate positive for EBV. Her lung EBV-lymphoproliferative disease was treated with rituximab. Parents were first cousins and two other sisters also had a CD4 lymphopenia associated with opportunistic infections, both dying of infectious complications post-HSCT.

The patient was initially treated conservatively with prophylactic valaciclovir, cotrimoxazole, fluconazole. However, at the age of 12 years old she developed an extra-nodal Bcell lymphoma within her left submaxillary salivary gland with evidence of paraprotein bands in her blood. Whole exome sequencing confirming a STK4 c.442C>T gene variant in the patient and both her affected siblings. Parents were heterozygous carriers. Because of the progressive disease, the patient underwent matched-sibling HSCT. Post-transplant course was complicated by CMV reactivation with diarrhea, which responded to ganciclovir. The patient is currently well four years post-HCST. 
Patient 7 A Turkish Cypriot boy presented at the age of 20 months old with a history of recurrent cough, wheeze and progressive dyspnea, which started at 14 months old. He had been admitted to hospital five times, once to the pediatric intensive care with "asthma", which only partly responded to inhaled bronchodilators and short courses of oral prednisolone. There was no consanguinity or significant family history.

Blood tests showed persistent lymphopenia $\left(0.5 \times 10^{9} / \mathrm{L}\right)$ with $\mathrm{CD} 3^{\text {low }} \mathrm{CD} 19^{\text {low }} \mathrm{CD} 56^{\text {normal }}$ lymphocyte subsets. Serum IgA was undetectable but IgM and IgG were normal, as were vaccine responses to tetanus, Haemophilus influenzae type B, measles and 4/12 pneumococcal serotypes. Chest CT scan showed extensive nodules in both lungs, particularly in the lower lobes. Respiratory secretions at bronchoalveolar lavage were positive for EBV by PCR. Lung biopsy showed a nodular, T-cell rich lymphohistiocytic infiltrate with EBV positivity demonstrated by in situ hybridization. A diagnosis of lung EBV-driven lymphoproliferative disease was made and the patient was treated with rituximab and EBV-directed cytotoxic Tlymphocyte infusions. Concurrently, the patient developed a rapidly progressive encephalopathy with hemiplegia and brain stem dysfunction requiring intensive care. Brain MR scan was indicative of progressive multifocal encephalopathy. Blood and urine BK, JC and astrovirus PCR were all negative. The patient deteriorated and died at the age of 21 months old. Postmortem was refused. Genetic results received after death showed a homozygous RAG1 c. $2333 \mathrm{G}>\mathrm{A}$ variant. Both parents were heterozygous carriers.

Patient 8 A Pakistani boy presented at the age of four years old with an eight-week history of fevers and weight loss and hepatomegaly. Chest $\mathrm{x}$-ray showed extensive consolidation and chest CT scan showed diffuse interstitial changes with fine reticulation and small nodular opacities in all lobes, particularly the right upper lobe, middle lobes and left lower lobes. There was no bronchiectasis. He was initially treated for tuberculosis, although there was no evidence of TB on culture, Mantoux or Quantiferon testing. EBV was identified by PCR from his sputum (log 

4.5) and blood ( $\log 4.1)$, although EBV IgM and IgG were both negative. Lymphocyte subsets were normal and serum immunoglobulins were all raised. Whole exome sequencing was performed and no variants in known genes caused PID was revealed, but a potentially pathogenic mutation in a novel gene has been identified and functional tests are ongoing.

The EBV lymphoproliferative disease was successfully treated with rituximab, and thus although lung biopsy had been considered to rule out lymphoma it was not performed. He underwent a matched unrelated HSCT and remains well and asymptomatic two years posttransplantation.

Patient 9 A previously well white European male presented in 2005 at the age of 14 years old with night sweats, vomiting, anorexia and weight loss but no diarrhea. There was no consanguinity or significant family history. An episode of chickenpox at three years old was no more severe than normal. Endoscopy showed active gastritis (Helicobacter pylori negative) with no evidence of inflammatory bowel disease. At 15 years old, following viral respiratory illness (which other family members also suffered), he developed a cough, progressive breathlessness, bilateral pleural effusions and respiratory failure. Laboratory investigations showed a generalized lymphopenia $\left(0.53 \times 10^{9} / \mathrm{L}\right)$, thrombocytopenia and hyperferritinemia $(5,636 \mu \mathrm{g} / \mathrm{L})$, but normal mitogen responses, serum immunoglobulin concentrations, ACE and neutrophil oxidative burst test.

Chest CT scan showed extensive reticulonodular interstitial shadowing, particularly in both lower lobes, with relative sparing of the upper lobes. Lung biopsy demonstrated an intraalveolar histiocytic and T-cell infiltrate with follicle formation. There were no granulomata. EBV, fungi and acid-fast bacilli were absent.

His lung disease did not respond to pulse methylprednisolone or methotrexate. He was ventilated in intensive care, but died one week later from progressive lung failure. Post-mortem histology 
182 of the lungs showed extensive infiltration of macrophages and lymphocytes, with diffuse

183 alveolar damage and hemorrhage. There was no evidence of infection, vasculitis or granuloma.

184 No EBV or CMV, or acid-fast bacilli were seen. Gut tissue showed no evidence of inflammatory

185 bowel disease. Bone (rib), lung, liver and lymph nodes all show prominent histiocytes with

186 hemophagocytosis. Candidate ADA/PNP, XAP and FHLH gene screen was normal, and

187 unfortunately there was insufficient DNA available to conduct whole exome sequencing after the 188 patient's death. 
189 Figure legends

190 Figure S1 Chest high resolution CT scans

191 See clinical case vignettes for description of pathology. 
1 Life-threatening pulmonary interstitial lung disease complicating pediatric non-

2 humoral immunodeficiencies

3

4 Tariq Al Farsi ${ }^{1}$ MD, Stephen M Hughes ${ }^{1}$ MB PhD, Robert F. Wynn ${ }^{2}$ MD, Edmund

5 Cheesman $^{3}$ MD, Frederic Rieux-Laucat ${ }^{4}$ PhD, Sylvain Latour ${ }^{4}$ PhD, Capucine Picard ${ }^{4,5}$ MD,

$6 \quad$ PhD, Sophie Hambleton ${ }^{1,6}$ MD, PhD, Peter D. Arkwright ${ }^{1}$ MB D Phil

7

$8{ }^{1}$ University of Manchester, Department of Paediatric Allergy \& Immunology, Manchester,

9 United Kingdom; ${ }^{2}$ University of Manchester, Department of Paediatric Haematology,

$10{ }^{3}$ Department of Paediatric Histopathology, Royal Manchester Children's Hospital,

11 Manchester, United Kingdom; ${ }^{4}$ Imagine Institute, Immunology, Paris, France; ${ }^{5}$ Study Center

12 for Primary Immunodeficiencies, Necker-Enfants Malades Hospital, APHP, Paris, France;

$13{ }^{6}$ University of Newcastle, Newcastle upon Tyne, United Kingdom

Author for correspondence: Dr P D Arkwright, Senior Lecturer in Paediatric Immunology,

Department of Paediatric Allergy \& Immunology, Royal Manchester Children’s Hospital,

Oxford Rd., Manchester, M13 9WL, United Kingdom, Tel + 44161701 0678, email

peter.arkwright@nhs.net

There were no external sources of funding. None of the authors declare any conflict of interests in relation to this study. 
22 Clinical Implications: Interstitial Lung Disease (ILD) in children often indicates life-

23 threatening PID and has a poor prognosis without hematopoietic stem cell transplantation.

24 Lung biopsy often provides definitive pathological results that help to direct management 25 decisions. 
To the Editor,

Recurrent acute and chronic lung infections associated with bronchiectasis are a major cause of morbidity and mortality in patients with Primary Immunodeficiency Disorders (PID).${ }^{1-3}$ In adults, inflammatory Interstitial Lung Disease (ILD) not due to pyogenic infection is found in up to $20 \%$ of patients with Common Variable Immunodeficiency Disorders (CVID) and can be associated with progressive restrictive lung disease and shortened survival. ${ }^{4-5}$ High resolution chest CT scan is the gold standard imaging technique for diagnosing ILD. Where there is doubt about the diagnosis lung biopsy is recommended. ${ }^{1,2}$ Histology classically shows lymphocytic infiltrates and/or granuloma, which in adults with CVID is termed Granulomatous-Lymphocytic Interstitial Lung Disease (GLILD). ${ }^{6}$ Although there are reports of ILD associated with a number of monogenic PIDs including Cytotoxic T-LymphocyteAssociated protein 4 (CTLA4), Lipopolysaccharide (LPS)-Responsive and Beige-like Anchor protein (LRBA), Recombination-Activating Gene (RAG1) deficiencies, X-linked Inhibitor of Apoptosis Protein (XIAP) and Chronic Granulomatous Disease (CGD), ${ }^{2,7,8}$ there are no published series delineating the prevalence, clinical characteristics, histologic features and management of children with PID and ILD. This retrospective case series aimed to review the genotype and clinicopathological phenotype of children presenting to our tertiary pediatric primary immunodeficiency center at Royal Manchester Children's Hospital, Manchester, United Kingdom between 2005 and 2018 with ILD and PID who underwent detailed investigations including chest CT scans and in most cases lung biopsy. We hypothesized that ILD in children often indicates life-threatening primary immunodeficiency disease and has a poor prognosis without hematopoietic stem cell transplantation (HSCT).

The case note review was part of a Natural History and Causes of CVID study approved by the Local Research Ethics Committee (03/ST/016). The demography, immunology, histology and genetic screening of nine patients $(0.9 \%)$ with PID and ILD out 
of 1,034 new pediatric referrals to our service because of recurrent, unusual or serious infections or inflammatory diseases were collated (Table I, Figure 1, and online supplementary material). This series of patients includes all but one patient known to Departments of Paediatric Histopathology, Immunology and Respiratory Medicine who had lung biopsies which demonstrated ILD associated with lymphocytic infiltration and/or granuloma formation.

Seven of the nine patients were males. Seven were Asian. All presented with a chronic cough and breathlessness suggestive of recurrent chest infections or asthma. In all but one case in which there was a family history of PID, it was the onset of chest symptoms that prompted a search for an underlying immunodeficiency disorder. A genetic diagnosis was confirmed in seven patients. Three children had a primary neutrophil immunodeficiency (two p40phox Chronic Granulomatous Disease (CGD) and one G6PC3 deficiency), and four a primary T-cell immunodeficiency (RAG1, STK4, LRBA, ITK). Whole exome sequencing of an eighth patient with pulmonary EBV-driven LPD uncovered a potentially pathogenic variant in a gene involved in T-cell signalling and further experiments to confirm the functional relevance of this novel finding are in progress. The ninth patient died from pulmonary Hemophagocytosis LymphoHistiocytosis (HLH) in 2006. Limited candidate gene screening at the time was negative and there was insufficient DNA available for subsequent whole exome sequencing. The median age of onset of chest symptoms of the nine patients was 12 months old. Except for one male who presented in the second year of life with RAG1 deficient Severe Combined Immunodeficiency (SCID) and died of progressive multifocal leukoencephalopathy, and a teenage boy who died of fulminant pulmonary HLH, the remaining seven patients were alive 12 months to 14 years post-HSCT.

Lung biopsies assisted to direct clinical management (Figure 1). Tuberculosis and fungal infections were excluded, allowing for the discontinuation of anti-microbials. No 
evidence of vasculitis was found in any of the patient's biopsies. Although granulomata were only seen in the patients with CGD, a number of the patients had a prominent histiocytic (CD68) infiltrate. This is in keeping with the lung histology of GLILD in adults with CVID, which is characterized by loose, more diffuse collections of histiocytic and lymphocytic infiltrates rather than well-defined clusters of histiocytes and multinuclear giant cells typical of sarcoidosis. ${ }^{5}$ In the three patients with primary neutrophil immunodeficiencies and the patient with the LRBA deficiency, lung biopsy confirmed the diagnosis of an inflammatory IBD rather than pyogenic infection, and responded to high dose corticosteroids prior to HSCT. In the four patients with primary T-cell immunodeficiencies, lung biopsies confirmed EBV-driven LPD, allowing focused treatment with rituximab \pm EBV-directed cytotoxic $T$ cells or chemotherapy. The lung biopsies of the teenager who died showed a mixed histiocytic, T- and B-lymphocytic infiltrate and evidence of hemophagocytosis. Infection, including EBV-driven LPD and vasculitis were excluded.

In conclusion, this is the first case series to delineate the variation in genotype and the frequent life-threatening clinical phenotype of children with ILD complicating PID. Lung biopsy often provides important information that helps to direct management decisions, not only by ruling out fungal and mycobacterial infections and vasculitis, but also in tailoring therapy based on the presence of inflammatory infiltrates or EBV-driven LPD. Diagnosis of the underlying PID is challenging, as patients may not only present with classical phenotypes such as EBV-positive ILD secondary to ITK deficiency, but also with unusual manifestations, for example "late-onset" SCID, or p40phox CGD where the standard dihydrorhodamine test may be normal. ${ }^{8,9}$ HSCT can be life-saving and should be considered as a definitive, curative therapeutic option. It is however important that HSCT is carried out by experienced transplant physicians, as the post-transplant course can be complicated by severe inflammation of the lungs, liver and gut in this group of patients. 
102 Acknowledgements

103 The authors are grateful to colleagues in the Departments of Histopathology at Great Ormond 104 St Hospital for Sick Children, London and the Royal Brompton Hospital, London for 105 reviewing atypical cases. Drs Latour, Picard and Rieux-Laucat provided the genetic diagnosis 106 for a number of the complex patients described in this report and helped draft the manuscript. 


\section{References}

108

1. Cinetto F, Scarpa R, Rattazzi M, Agostini C. The broad spectrum of lung diseases in primary antibody deficiencies. Eur Respir Rev. 2018;27:149.

2. Baumann U, Routes JM, Soler-Palacín P, Jolles S. The Lung in Primary Immunodeficiencies: New Concepts in Infection and Inflammation. Front Immunol. 2018;9:1837.

3. Jesenak M, Banovcin P, Jesenakova B, Babusikova E. Pulmonary manifestations of primary immunodeficiency disorders in children. Front Pediatr. 2014;2:77.

4. Hartono S, Motosue MS, Khan S, Rodriguez V, Iyer VN, Divekar R, et al. Predictors of granulomatous lymphocytic interstitial lung disease in common variable immunodeficiency. Ann Allergy Asthma Immunol. 2017;118:614-620.

5. Bates CA, Ellison MC, Lynch DA, Cool CD, Brown KK, Routes JM. Granulomatouslymphocytic lung disease shortens survival in common variable immunodeficiency. J Allergy Clin Immunol. 2004;114:415-21.

6. Hurst JR, Verma N, Lowe D, Baxendale HE, Jolles S, Kelleher P, et al. British Lung Foundation/United Kingdom Primary Immunodeficiency Network Consensus Statement on the Definition, Diagnosis, and Management of Granulomatous-Lymphocytic Interstitial Lung Disease in Common Variable Immunodeficiency Disorders. J Allergy Clin Immunol Pract. 2017;5:938-945.

7. Steele CL, Doré M, Ammann S, Loughrey M, Montero A, Burns SO, et al. X-linked Inhibitor of Apoptosis complicated by Granulomatous Lymphocytic Interstitial Lung Disease (GLILD) and granulomatous hepatitis. J Clin Immunol. 2016;36:733-8.

8. van de Geer A, Nieto-Patlán A, Kuhns DB, Tool AT, Arias AA, Bouaziz M, et al. Inherited p40phox deficiency differs from classic chronic granulomatous disease. J Clin Invest. 2018;128:3957-3975. 
135

136 Figure 1 Characteristic lung biopsies of different PID associated with interstitial lung

137 disease. Panels (top to bottom) show Hematoxylin \& Eosin (H\&E), CD3 (T-cells), CD20 (B-

138 cells), CD68 (histiocytes), EBV in situ hybridization (EBV) for each condition. 


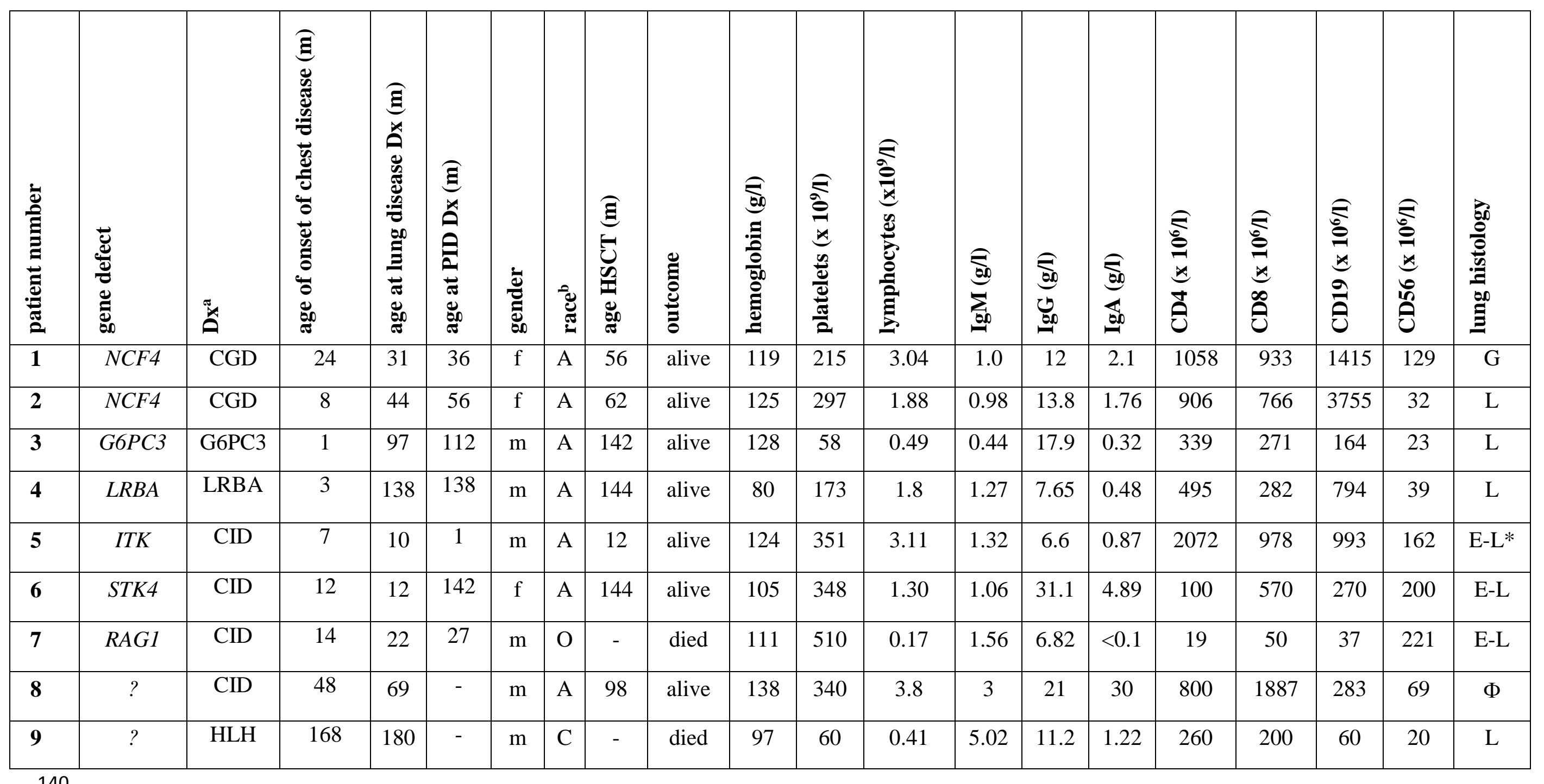


141 Dx: diagnosis, PID: primary immunodeficiency disease, NCF4: Neutrophil Cytosolic Factor 4, ITK: IL-2 Inducible T-Cell Kinase, STK4:

142 Serine/Threonine Kinase 4, RAG1: Recombination Activating Gene 1, aCGD: chronic granulomatous disease, G6PC3: Glucose-6-Phosphatase

143 Catalytic Subunit 3 deficiency, LRBA: lipopolysaccharide (LPS)-responsive and beige-like anchor protein deficiency, CID: combined T/B-cell

144 immunodeficiency, ${ }^{\mathrm{b}} \mathrm{C}$ : Caucasian A: Asian, O: Other. Lung histology: non-caseating granuloma (G), lymphoid hyperplasia (L), lymphoma (L*),

$145 \mathrm{EBV}(\mathrm{E}), \Phi$ - EBV-lymphoproliferative disease diagnosed after finding EBV from sputum, nodular shadowing on CT scan images and response

146 to rituximab. 


\section{Online material}

2 Life-threatening pulmonary interstitial lung disease complicating pediatric non-humoral 3 immunodeficiencies

$4 \quad$ Tariq Al Farsi ${ }^{1}$ MD, Stephen M Hughes ${ }^{1}$ MB PhD, Robert F. Wynn ${ }^{2}$ MD, Edmund Cheesman ${ }^{3}$ 5 MD, Frederic Rieux-Laucat ${ }^{4}$ PhD, Sylvain Latour ${ }^{4}$ PhD, Capucine Picard ${ }^{4,5}$ MD, PhD, Sophie $6 \quad$ Hambleton $^{6}$ MD, PhD, Peter D. Arkwright ${ }^{1}$ MB D Phil

$8{ }^{1}$ University of Manchester, Department of Paediatric Allergy \& Immunology, Manchester,

9 United Kingdom; ${ }^{2}$ University of Manchester, Department of Paediatric Haematology,

$10{ }^{3}$ Department of Paediatric Histopathology, Royal Manchester Children's Hospital, Manchester, 11 United Kingdom; ${ }^{4}$ Imagine Institute, Immunology, Paris, France; ${ }^{5}$ Study Center for Primary 12 Immunodeficiencies, Necker-Enfants Malades Hospital, APHP, Paris, France; ${ }^{6}$ University of 13 Newcastle, Newcastle upon Tyne, United Kingdom 
Clinical case vignettes (high resolution chest CT scans are shown in Figure S1)

Patient 1 A four-year-old Pakistani girl presented at the age of two years old with a chronic cough and clubbing, associated with cervical lymphadenopathy and hepatosplenomegaly. Parents were first cousins. There was no family history of chest problems or tuberculosis. A chest x-ray showed bilateral, multinodular shadowing. Chest CT scan confirmed patchy infiltrates in the right and left lower lobes, but no bronchiectasis. Hilar lymphadenopathy was noted, particularly on the right $(7 \times 10 \mathrm{~mm})$. Bronchioalveolar lavage revealed no microbes. Lymph node biopsy showed granulomatous inflammation without caseation. Stains for mycobacteria and fungi were negative. Because of the chronic unremitting nature of the lung disease a lung biopsy was performed, which showed granulomatous lymphocytic infiltration with no organisms identified.

Standard dihydrorhodamine test with PMA was normal, but stimulation with E. coli was reduced. Gene sequencing revealed a homozygous c. $118-1 \mathrm{G}>\mathrm{A}$ splice variant in the NCF4 gene consistent with p40phox CGD. ${ }^{8}$ Parents were both heterozygous carriers. The symptoms did not respond to anti-microbials and could only partly be controlled with intermittent pulse intravenous methylprednisolone.

In view of the diagnosis of CGD and her ongoing inflammatory lung disease, she underwent matched-sibling HSCT at the age of three years old. Post-transplant course was complicated by mild veno-occlusive disease treated with defibrotide, followed by fulminant inflammatory disease of her gut, liver and lungs, complicated by pulmonary hemorrhage, requiring high frequency oscillatory ventilation in intensive care. The lung disease was brought under control with anti-TNF therapy and extra-corporal photopheresis. Twelve months posttransplant she developed autoimmune thrombocytopenia, which responded to rituximab. 
Eighteen months post-transplant, her condition is stable and systemic immune suppression has been discontinued. Peripheral leukocytes are $100 \%$ donor.

Patient 2 A seven-year-old Pakistani girl presented with a history of chronic chest symptoms going back to when she was eight months old. She had a chest deformity, clubbing and oxygen saturations in air of $88 \%$. Her growth and development were delayed and she had intermittent fevers and an iron deficiency anemia. Parents were first cousins. No pathogens had been isolated from her chest. Because of wheeze and breathlessness, she had been treated with recurrent courses of oral prednisolone. Chest x-ray and CT scan showed hilar lymphadenopathy and bilateral patchy shadowing and nodules especially in both lower lobes. There was no bronchiectasis. Lung biopsy showed interstitial inflammation with lymphoid follicle formation. There was alveolar remodelling, prominent type 2 pneumocyte hyperplasia and abundant intraalveolar proteinaceous exudates.

Dihydrorhodamine test with PMA was normal, but stimulation with E. coli was reduced. Gene sequencing revealed a homozygous c. $32+2 \mathrm{~T}>\mathrm{G}$ variant in $N C F 4$ consistent with $\mathrm{p} 40 \mathrm{phox}$ CGD. Both parents were found to be carriers. Her inflammatory lung disease was only partly controlled with pulse methylprednisolone therapy. She therefore underwent matched unrelated HSCT at the age of five years old. Three months post-transplantation, she developed lung EBV LPD, which was treated with rituximab. Four months post-transplant she developed a central line infection complicated by Klebsiella pneumoniae septicemia, cardiac tamponade and cardiogenic shock requiring intensive care. Two years post transplantation she is now well and off all medication.

Patient 3 A 12-year-old Pakistani boy suffered from episodes of cough, wheeze and breathlessness from one month of age. He had been treated with multiple courses of antibiotics, inhaled steroids, nebulized bronchodilators and oral prednisolone. He also had chronically loose 
stools. His parents were first cousins. He was referred to our primary immunodeficiency centre at the age of nine years old after moving to the UK. He had been diagnosed with a congenital neutropenia at the age of six years and started on regular G-CSF, although continued to have persistent cough and dyspnea, with poor exercise tolerance requiring supplementary night time oxygen. $\mathrm{FEV}_{1}$ and FVC were $30 \%$ of predicted. Stool culture, bronchoscopy and bronchoalveolar lavage revealed no pathogens. Mantoux and Quantiferon tests were negative.

High resolution chest CT scan showed air trapping and nodular opacities in the left lower lobe, with only mild basal bronchiectasis. Lung biopsy showed peri-bronchial mononuclear infiltrate with occasional lymphoid nodules with no granuloma, fungi or mycobacteria. There was no fibrosis or pulmonary hypertensive changes. Gut biopsies showed inflammation with cryptitis, crypt abscesses, inflammatory infiltrate throughout the colon but no granuloma. There were also patchy inflammatory changes in the esophagus, stomach and terminal ileum.

In view of his congenital neutropenia, symmetrical growth retardation, cryptorchidism and distended veins on his anterior chest, genetic testing was performed which confirmed a homozygous frameshift c882_903 duplication in the G6PC3 gene. Because of frequent hospital admissions for his lung disease and poorly controlled inflammatory bowel disease he underwent matched unrelated HSCT. Fifteen months post-transplant, he is now able to scooter along the flat and not needing supplementary oxygen. His stool frequency has also decreased. Post-transplant immune suppression has been discontinued with $100 \%$ donor engraftment.

Patient 4 An eleven-year-old Indian boy presented in infancy with chronic watery diarrhea due to an autoimmune non-granulomatous colitis. He was treated for four years with corticosteroids, to which azathioprine was added. His parents were first cousins. At seven years old he developed generalized atopic dermatitis and at eight years old cervical lymphadenopathy, splenomegaly, chronic urticaria, a Coombs positive hemolytic anemia and ITP with epistaxis, 
which only partly responded to corticosteroids, methotrexate and IV rituximab. At the age of ten years old, while on the methotrexate (10mg/m²/week), he developed ILD (EBV positive by PCR on sputum but not blood) with fevers and signs of respiratory distress. High resolution chest CT scan showed confluent dense consolidation in both lower lobes, multiple areas of hazy nodular, ground glass changes and hilar lymphadenopathy. Lung biopsy showed a diffuse, reactive lymphoid hyperplasia with lymphoid follicle formation. At the same time his autoimmune cytopenias became more difficult to control. His lung disease and cytopenias only partly responded to rituximab, and required high dose systemic corticosteroids.

Whole exome sequencing revealed a homozygous c.5770_5776 deletion in the LRBA gene. Both parents were carriers. As there were no suitable matched sibling or unrelated donors, he underwent a maternal haploidentical peripheral blood TCR $\alpha \beta$-depleted HSCT with reduced intensity conditioning. His post-transplant course was uncomplicated. Four years posttransplant he off all medication and has $100 \%$ donor engraftment. His chest disease, autoimmune enteropathy and cytopenias have resolved. Because of proteinuria, he investigated and diagnosed with diffuse mesangial sclerosis, which is currently not progressive and he has normal renal function and no proteinuria.

Patient 5 Prompted by a family history, a 15-year-old boy diagnosed with a homozygous c.626G > A ITK deficiency at the age of one month old. HSCT was also delayed because of Hirschsprung's disease requiring surgery in the neonatal period and the difficulty finding a suitable donor. At the age of seven months, EBV viremia was noted and chest CT scan showed widespread bulky masses throughout both lungs. The largest of these replaced most of the right upper lobe. There were multiple smaller nodules scattered throughout both lungs. Lung biopsy showed replacement of normal parenchyma with an EBV-driven diffuse large B-cell lymphoma. He was treated with rituximab and UK CCSG NHL901 chemotherapy protocol, 
followed by a single allelic A mismatched related donor transplant. He remains well off all therapy 14 years post HSCT.

Patient 6 A 16-year-old Asian girl presented at the age of three years old with a history of recurrent infections from 14 months old, when she developed viral croup requiring ventilation and cryptosporidial diarrhea. Over the next few years, she suffered from recurrent Streptococcus pneumoniae pneumonia and ear infections, as well as recurrent thrush, molluscum contagiosum and herpes gingivostomatitis requiring intravenous acyclovir. She had a chronic productive cough, which did not respond to asthma inhalers. Examination revealed tachypnea and oxygen saturations in air of $94 \%$, a discharging left ear and oral thrush.

Investigations showed a CD4 lymphopenia but raised, reactive serum immunoglobulins and protective vaccine antibody responses. HIV screen was negative. EBV was detected in the blood by PCR. Chest CT scan showed multiple small nodules of different sizes throughout both lungs and no bronchiectasis. Lung biopsy showed a B-cell infiltrate positive for EBV. Her lung EBV-lymphoproliferative disease was treated with rituximab. Parents were first cousins and two other sisters also had a CD4 lymphopenia associated with opportunistic infections, both dying of infectious complications post-HSCT.

The patient was initially treated conservatively with prophylactic valaciclovir, cotrimoxazole, fluconazole. However, at the age of 12 years old she developed an extra-nodal Bcell lymphoma within her left submaxillary salivary gland with evidence of paraprotein bands in her blood. Whole exome sequencing confirming a STK4 c.442C>T gene variant in the patient and both her affected siblings. Parents were heterozygous carriers. Because of the progressive disease, the patient underwent matched-sibling HSCT. Post-transplant course was complicated by CMV reactivation with diarrhea, which responded to ganciclovir. The patient is currently well four years post-HCST. 
Patient 7 A Turkish Cypriot boy presented at the age of 20 months old with a history of recurrent cough, wheeze and progressive dyspnea, which started at 14 months old. He had been admitted to hospital five times, once to the pediatric intensive care with "asthma", which only partly responded to inhaled bronchodilators and short courses of oral prednisolone. There was no consanguinity or significant family history.

Blood tests showed persistent lymphopenia $\left(0.5 \times 10^{9} / \mathrm{L}\right)$ with $\mathrm{CD} 3^{\text {low }} \mathrm{CD} 19^{\text {low }} \mathrm{CD} 56^{\text {normal }}$ lymphocyte subsets. Serum IgA was undetectable but IgM and IgG were normal, as were vaccine responses to tetanus, Haemophilus influenzae type B, measles and 4/12 pneumococcal serotypes. Chest CT scan showed extensive nodules in both lungs, particularly in the lower lobes. Respiratory secretions at bronchoalveolar lavage were positive for EBV by PCR. Lung biopsy showed a nodular, T-cell rich lymphohistiocytic infiltrate with EBV positivity demonstrated by in situ hybridization. A diagnosis of lung EBV-driven lymphoproliferative disease was made and the patient was treated with rituximab and EBV-directed cytotoxic Tlymphocyte infusions. Concurrently, the patient developed a rapidly progressive encephalopathy with hemiplegia and brain stem dysfunction requiring intensive care. Brain MR scan was indicative of progressive multifocal encephalopathy. Blood and urine BK, JC and astrovirus PCR were all negative. The patient deteriorated and died at the age of 21 months old. Postmortem was refused. Genetic results received after death showed a homozygous RAG1 c. $2333 \mathrm{G}>\mathrm{A}$ variant. Both parents were heterozygous carriers.

Patient 8 A Pakistani boy presented at the age of four years old with an eight-week history of fevers and weight loss and hepatomegaly. Chest $\mathrm{x}$-ray showed extensive consolidation and chest CT scan showed diffuse interstitial changes with fine reticulation and small nodular opacities in all lobes, particularly the right upper lobe, middle lobes and left lower lobes. There was no bronchiectasis. He was initially treated for tuberculosis, although there was no evidence of TB on culture, Mantoux or Quantiferon testing. EBV was identified by PCR from his sputum (log 

4.5) and blood ( $\log 4.1)$, although EBV IgM and IgG were both negative. Lymphocyte subsets were normal and serum immunoglobulins were all raised. Whole exome sequencing was performed and no variants in known genes caused PID was revealed, but a potentially pathogenic mutation in a novel gene has been identified and functional tests are ongoing.

The EBV lymphoproliferative disease was successfully treated with rituximab, and thus although lung biopsy had been considered to rule out lymphoma it was not performed. He underwent a matched unrelated HSCT and remains well and asymptomatic two years posttransplantation.

Patient 9 A previously well white European male presented in 2005 at the age of 14 years old with night sweats, vomiting, anorexia and weight loss but no diarrhea. There was no consanguinity or significant family history. An episode of chickenpox at three years old was no more severe than normal. Endoscopy showed active gastritis (Helicobacter pylori negative) with no evidence of inflammatory bowel disease. At 15 years old, following viral respiratory illness (which other family members also suffered), he developed a cough, progressive breathlessness, bilateral pleural effusions and respiratory failure. Laboratory investigations showed a generalized lymphopenia $\left(0.53 \times 10^{9} / \mathrm{L}\right)$, thrombocytopenia and hyperferritinemia $(5,636 \mu \mathrm{g} / \mathrm{L})$, but normal mitogen responses, serum immunoglobulin concentrations, ACE and neutrophil oxidative burst test.

Chest CT scan showed extensive reticulonodular interstitial shadowing, particularly in both lower lobes, with relative sparing of the upper lobes. Lung biopsy demonstrated an intraalveolar histiocytic and T-cell infiltrate with follicle formation. There were no granulomata. EBV, fungi and acid-fast bacilli were absent.

His lung disease did not respond to pulse methylprednisolone or methotrexate. He was ventilated in intensive care, but died one week later from progressive lung failure. Post-mortem histology 
182 of the lungs showed extensive infiltration of macrophages and lymphocytes, with diffuse

183 alveolar damage and hemorrhage. There was no evidence of infection, vasculitis or granuloma.

184 No EBV or CMV, or acid-fast bacilli were seen. Gut tissue showed no evidence of inflammatory

185 bowel disease. Bone (rib), lung, liver and lymph nodes all show prominent histiocytes with

186 hemophagocytosis. Candidate ADA/PNP, XAP and FHLH gene screen was normal, and

187 unfortunately there was insufficient DNA available to conduct whole exome sequencing after the 188 patient's death. 
189 Figure legends

190 Figure S1 Chest high resolution CT scans

191 See clinical case vignettes for description of pathology. 
Figure No. 1 - Unmarked

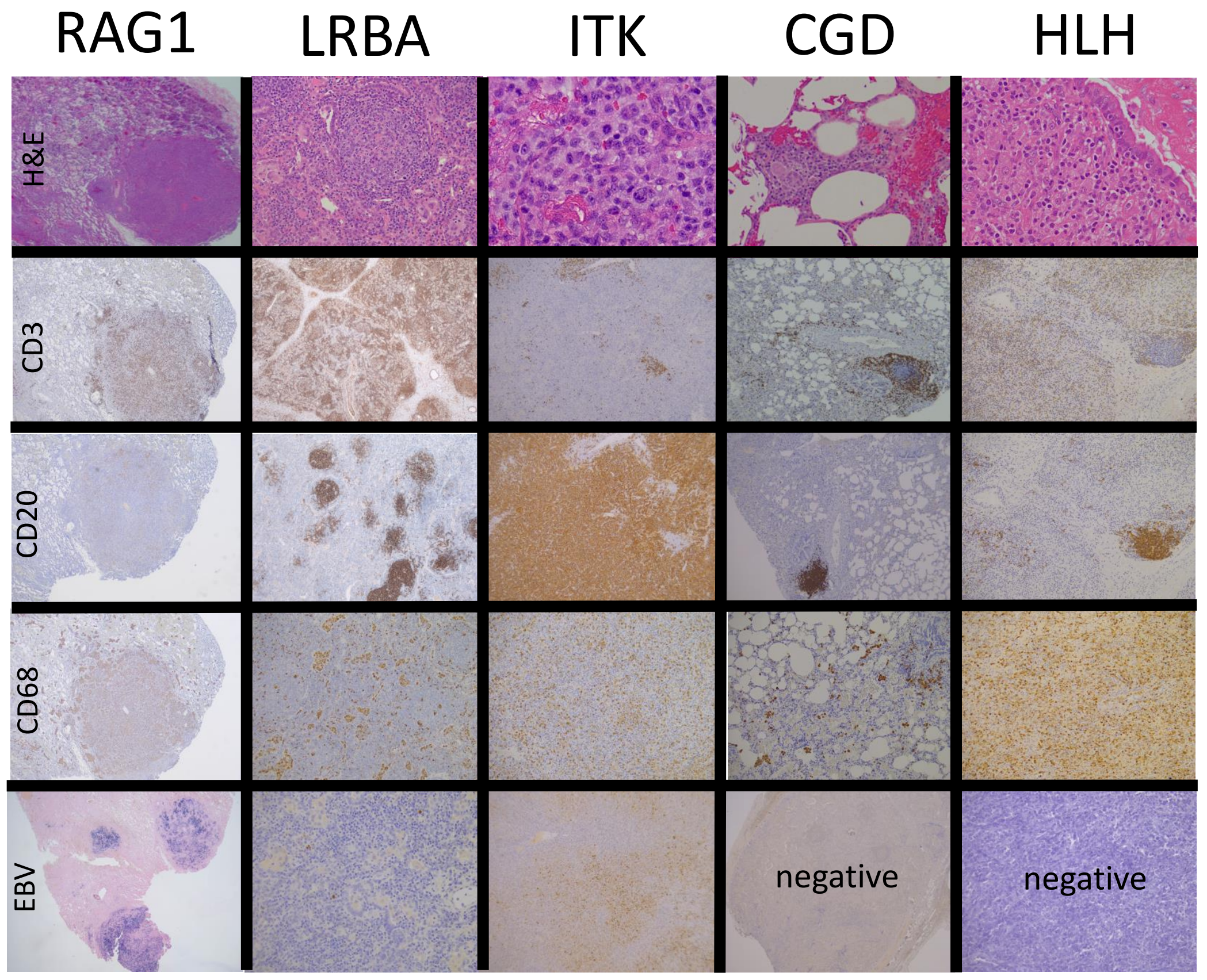

\section{RAG1}

LRBA

ITK

CGD

HLH 

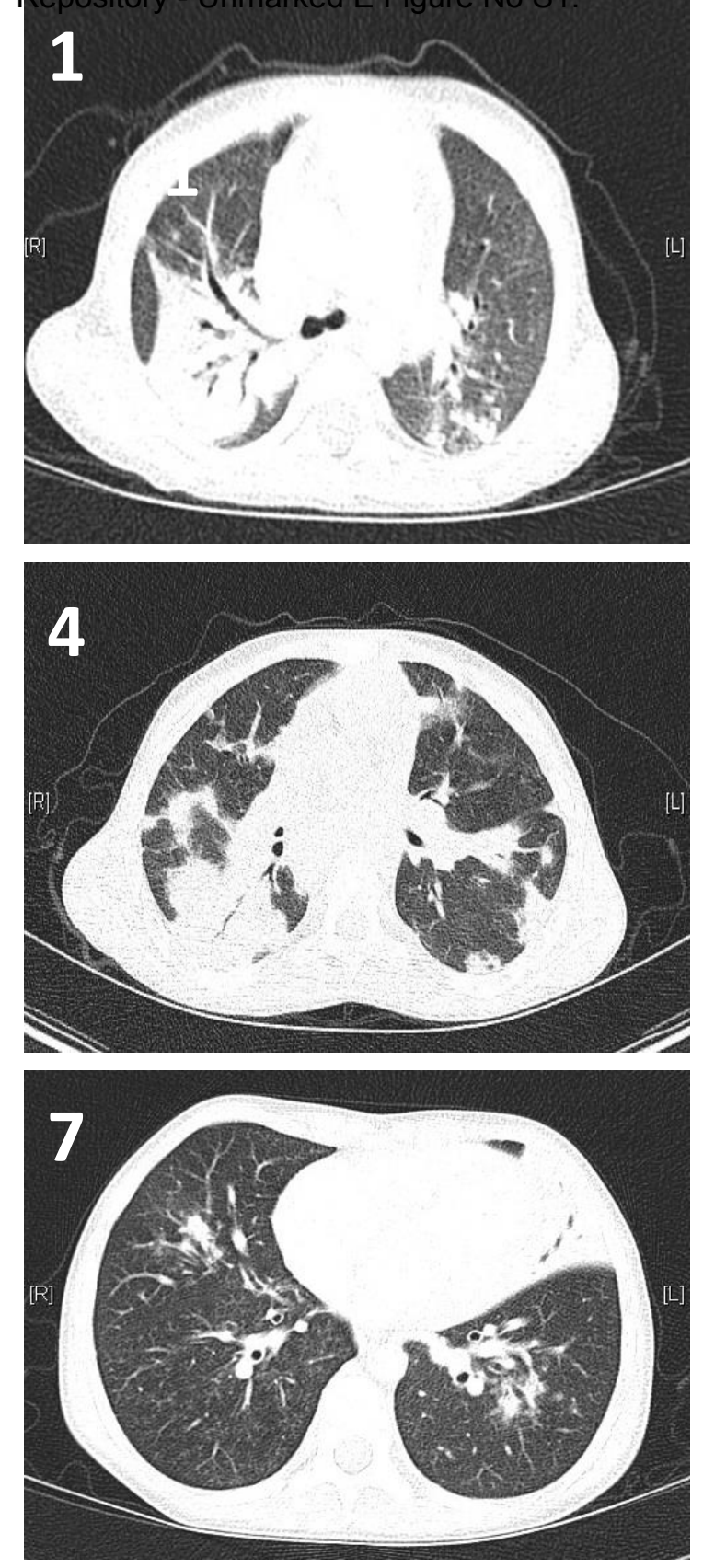
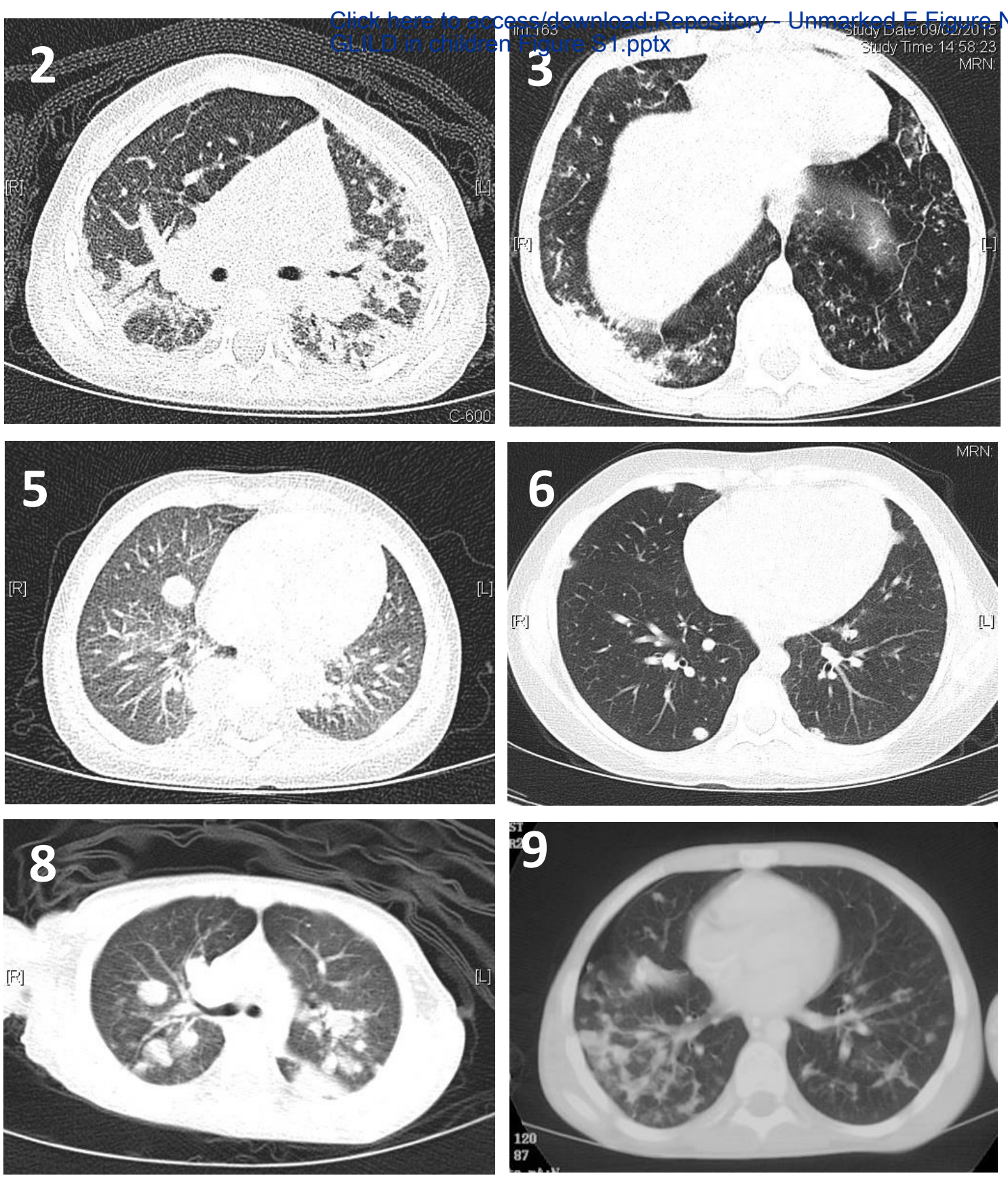